\title{
Globe
}

Revue internationale d'études québécoises

\section{Manuel Feifel : Regionen als " Global Players ". Das Beispiel der interregionalen Kooperation Bayern-Québec. Synchron, Heidellberg, 2003}

\section{Manuel Meune}

Volume 7, numéro 2, 2004

URI : https://id.erudit.org/iderudit/1000868ar

DOI : https://doi.org/10.7202/1000868ar

Aller au sommaire du numéro

Éditeur(s)

Globe, Revue internationale d'études québécoises

ISSN

1481-5869 (imprimé)

1923-8231 (numérique)

Découvrir la revue

Citer ce compte rendu

Meune, M. (2004). Compte rendu de [Manuel Feifel : Regionen als « Global

Players ». Das Beispiel der interregionalen Kooperation Bayern-Québec.

Synchron, Heidelberg, 2003]. Globe, 7(2), 199-202.

https://doi.org/10.7202/1000868ar d'utilisation que vous pouvez consulter en ligne.

https://apropos.erudit.org/fr/usagers/politique-dutilisation/ 
RECENSIONS

\section{Manuel Feifel}

Regionen als "Global Players". Das Beispiel der interregionalen Kooperation Bayern-Québec.

\section{Synchron, Heidelberg, 2003.}

Dans cet ouvrage, Manuel Feifel, qui a suivi un parcours universitaire en relations internationales et en gestion culturelle, aborde d'une façon inhabituelle la spécificité québécoise, par le biais de la "coopération interrégionale * entre le Québec et la Bavière. Le lecteur québécois qui sourcillerait à la lecture du terme "région ", appliqué au Québec, sera rassuré par la préface d'Ingo Kolboom, fin spécialiste d'un Québec qu'il n'a jamais hésité à étudier sous l'angle national. Mais le livre est bel et bien une invitation à ne pas ramener la politique extérieure québécoise à une querelle avec Ottawa, et à la situer plutôt dans une dynamique plus large. Dans sa partie théorique, l'auteur insiste sur la complémentarité dialectique entre "régionalisation " et "mondialisation * et il analyse la différence entre les entités qui disposent d'un statut officiel d'État souverain, et celles qui, à l'image du Québec et de la Bavière, découvrent une marge de manœuvre jadis dévolue aux "vrais " États-nations, en faisant l'expérience de " relations transfédérales ".

Après un bref aperçu de l'histoire des deux sociétés, Feifel passe en revue les points qu'elles ont en commun et qui vont bien au-delà de la réputation qu'elles partagent dans leur cadre politique respectif en tant qu'entités rebelles, à savoir une intégration continentale (ALENA, UE), une structure économique (passage rapide d'une économie agraire à une société hautement technologisée), une identité forte (catholicisme ; langue - dialecte régional dans le cas du bavarois) et un certain ancrage historique. Une autre similitude serait, selon l'auteur, un lien privilégié avec la France, c'est-à-dire, dans le cas de la Bavière, le rôle de la France dans la naissance de l'État bavarois moderne, la visite du général de Gaulle en 1962 ou encore les nombreux jumelages avec des villes françaises.

Feifel illustre ensuite les paradoxes des systèmes fédéraux allemand et canadien : le Canada apparaît unitariste malgré sa profession de foi confédérale, puisqu'il n'a pas ancré dans sa constitution l'autonomie des provinces, à l'inverse de l'Allemagne, souvent présentée comme une fédération assez rigide, mais dont les länder, très autonomes, disposent d'une constitution. Alors que la loi fondamentale de l'Allemagne précise 
qu'un land peut conclure des traités internationaux, la situation du Québec fait l'objet d'interprétations contradictoires : la constitution de 1867 ne stipulant pas que les relations extérieures sont de juridiction fédérale, le Québec considère qu'elles relèvent de ses propres compétences, ce que l'État fédéral tend cependant à contester. Feifel démontre que pour l'état central allemand, les activités extérieures de la Bavière ne posent pas de problèmes et s'effectuent sur le mode de la coopération harmonieuse, tandis que celles du Québec ont plutôt été entravées par Ottawa.

C'est dans un discours de Lucien Bouchard prononcé en 1999 que Feifel décèle la progression d'une acception inhabituelle du terme "région ", qui relève d'une nouvelle perception des échanges internationaux ne contredisant pas la perspective nationale. La Bavière, qui ne se présente pas comme une nation, souhaite néanmoins amplifier la décentralisation, alors que Québec jongle avec l'idée de l'indépendance nationale ; pourtant, Feifel rappelle que ces variations sur le thème de la souveraineté n'empêchent aucunement la bonne entente entre deux entités qui parient, aussi, sur l'ouverture des frontières.

Feifel distingue cinq périodes dans la coopération québécobavaroise. Pendant la phase de " gestation " (1983-1989), Bernard Landry, alors ministre du commerce extérieur, trouve en Franz-Josef Strauss, Premier ministre francophile, un interlocuteur de choix. Il visite avec bonheur l'، État libre * de Bavière en 1983 et accueille Strauss un an plus tard, à une époque où le land de Rhénanie du Nord-Westphalie, qui abrite depuis 1970 un bureau économique du Québec à Düsseldorf, ne se montre guère intéressé à cultiver des liens politiques avec le gouvernement québécois. Pendant la période de "formalisation " (1989-1993), le Premier ministre Robert Bourassa intensifie la coopération. La troisième période, celle de la "consolidation" (1993-1997), est marquée par les liens entre Bernard Landry, devenu Vice-premier ministre, et le Premier ministre Edmund Stoiber. Ce renforcement des liens est paradoxalement lié aux choix budgétaires du gouvernement Bouchard, qui entraînent la fermeture du bureau de Düsseldorf en 1996: la coopération québécobavaroise souffrant de l'absence de représentation du Québec en Allemagne, la Bavière vole alors au secours de son partenaire en mettant gracieusement des locaux à sa disposition; en 1997, le Québec ouvre une nouvelle représentation en Allemagne, à Munich cette fois et le rôleclé de la Bavière est scellé. Pendant la phase d'institutionnalisation, c'est 
au tour de la Bavière d'ouvrir un bureau - à vocation économique - à Montréal, en 1999, puis, deux ans plus tard, une représentation avec des prérogatives plus larges que les autres représentations bavaroises, notamment en matière de culture. Cette période est marquée par un discours de Stoiber à l'Assemblée nationale du Québec et par celui de Landry au parlement régional (Landtag) de Bavière en 2001. La dernière phase (à partir de 2002) est caractérisée par un élargissement multilatéral de la coopération vers la province sud-africaine du Cap-Occidental, la province chinoise du Shandong, la Haute-Autriche et la Californie. L'auteur pose enfin la question d'une éventuelle dilution de la coopération au-delà du rôle moteur qu'ils sont censés jouer.

Feifel examine en outre le détail des traités de coopération dans les domaines les plus variés : tourisme, formation, culture, environnement, santé, intelligence artificielle, multimédias, secteur aéronautique, etc. Il souligne les succès de la collaboration technique et économique - sauf en matière d'écologie, où les principes n'auraient pas été mis en application. Il apparaît qu'au fil des ans, la dimension culturelle a pris de l'ampleur, d'autant qu'au bureau du Québec à Munich sont venus s'ajouter des services culturels à Berlin - dont l'auteur, précisons-le, est le coresponsable. Dès 1993 des échanges d'élèves, d'assistants de langue et d'étudiants ont été mis en place. Des bourses ont permis à des Bavarois d'étudier au Québec, puis à des Québécois d'étudier en Bavière. Des échanges de journalistes ont amélioré la connaissance que chaque société pouvait avoir de l'autre. Des expositions ont vu le jour, ainsi que des collaborations dans les domaines de la danse, de la littérature, de la musique - en partie grâce à la Maison des artistes où peuvent séjourner des Québécois.

Si l'auteur estime positif le bilan des échanges culturels, il souligne toutefois la dissymétrie des échanges, puisque le Québec ne peut octroyer toutes les bourses d'étude, faute d'un assez grand nombre de candidats ayant une bonne connaissance de l'allemand. On touche ici aux limites de la coopération : l'enseignement des langues autres que l'anglais et le français dans les écoles secondaires et les CÉGEPS du Québec apparaît en effet très limité par rapport au système scolaire bavarois au sein duquel la plupart étudient au moins deux langues étrangères. Si les Bavarois parlant le français sont nombreux à souhaiter découvrir le Québec, les Québécois à l'aise en allemand sont encore trop rares. L'auteur rappelle par ailleurs que du côté bavarois, la gestion de 


\section{REVUE INTERNATIONALE D'ÉTUDES QUÉBÉCOISES}

la coopération incombe uniquement à la chancellerie d'État, tandis que le Québec dispose de son puissant ministère des Relations internationales, dont certains fonctionnaires regrettent de ne pas trouver en Bavière d'institution équivalente. Malgré ces insuffisances, Feifel souligne que le climat de confiance instauré est irréversible, et il formule le vœu que le Québec et la Bavière se dotent d'un site Internet plus performant - un site commun si possible -, qui rendrait accessible au grand public une documentation bilingue - mémoires, extraits de presse, etc.

Il est peu question, dans l'ouvrage de Manuel Feifel, des perceptions réciproques. L'auteur n'avait pas pour projet d'étudier l'image de la Bavière au Québec et celle du Québec en Bavière. Il serait toutefois souhaitable qu'une étude de l'évolution de ces perceptions interculturelles vienne un jour compléter le tableau très instructif qu'a brossé Feifel. Celui-ci est en tout cas parvenu à présenter avec un recul certain un sujet caractérisé par son extrême contemporanéité, et on espère que son ouvrage, riche en détails factuels et analyses très utiles, sera rapidement traduit en français. Au-delà de la question des liens entre la Bavière et le Québec, qui méritait qu'on lui consacre un ouvrage, le livre s'adresse, par la clarté de l'exposé théorique, à tous ceux qui s'intéressent aux "échanges interrégionaux ". L'auteur se demande d'ailleurs en conclusion si, après l'effritement des États-nations, les régions ne seront pas appelées à leur tour à perdre leur influence dans la foulée d'une mise en réseaux généralisée entre institutions et entreprises. Ajoutons pour terminer que l'ouvrage est accompagné d'un cédérom très bien documenté avec photographies, textes officiels en français et en allemand, transcriptions d'entrevues - et, en prime, d'un document audio qui permet de découvrir l'entretien que Bernard Landry a accordé à l'auteur.

Manuel Meune

Université de Montréal 